\title{
FUS mutations in sporadic amyotrophic lateral sclerosis: Clinical and genetic analysis
}

\author{
William Sproviero ${ }^{\mathrm{a}}$, Vincenzo La Bella ${ }^{\mathrm{b}}$, Rosalucia Mazzei ${ }^{\mathrm{a}}$, Paola Valentino ${ }^{\mathrm{c}}$, \\ Carmelo Rodolico ${ }^{\mathrm{d}}$, Isabella Laura Simone ${ }^{\mathrm{e}}$, Giancarlo Logroscino ${ }^{\mathrm{e}}$, Carmine Ungaro ${ }^{\mathrm{a}}$, \\ Angela Magariello ${ }^{\mathrm{a}}$, Alessandra Patitucci ${ }^{\mathrm{a}}$, Gioacchino Tedeschi ${ }^{\mathrm{f}}$, Rossella Spataro ${ }^{\mathrm{b}}$, \\ Francesca Condino $^{\mathrm{a}}$, Francesco Bono ${ }^{\mathrm{c}}$, Luigi Citrigno ${ }^{\mathrm{a}}$, Maria Rosaria Monsurrò ${ }^{\mathrm{f}}$, \\ Maria Muglia $^{\mathrm{a}}$, Antonio Gambardella ${ }^{\mathrm{a}, \mathrm{c}}$, Aldo Quattrone ${ }^{\mathrm{c}}$, Francesca Luisa Conforti ${ }^{\mathrm{a}, *}$ \\ ${ }^{a}$ Institute of Neurological Sciences, National Research Council, Mangone, Cosenza, Italy \\ ${ }^{b}$ ALS Clinical Research Center, Bio.Ne.C, University of Palermo, Italy \\ ${ }^{c}$ Institute of Neurology, University Magna Graecia, Catanzaro, Italy \\ ${ }^{d}$ Department of Neurosciences, Psychiatric and Anaesthesiological Sciences, University of Messina, Italy \\ ${ }^{e}$ Department of Neurological and Psychiatric Sciences, University of Bari, Italy \\ ${ }^{f}$ Second Division of Neurology, Second University of Naples, Naples, Italy
}

Received 23 May 2011; received in revised form 3 October 2011; accepted 4 October 2011

\begin{abstract}
Fused in sarcoma (FUS) or translocation in liposarcoma (TLS), a DNA/RNA-binding protein, causes a dominant autosomal inherited form of amyotrophic lateral sclerosis (ALS), ALS 6. Its main role in neurodegeneration is highlighted by the presence of cytoplasmic accumulation of its mutant protein form in ALS patients. To further define the frequency and spectrum of FUS gene mutations, we have performed a molecular screening of a cohort of 327 Italian patients from Southern Italy with sporadic ALS (SALS). We identified 4 patients carrying 3 different missense mutations and several polymorphisms. Two different substitutions occurring in the same amino acidic position have been observed in 2 patients: R521G and R521C respectively; P525L mutation has been found in 2 additional cases. Most of the patients with FUS mutations showed early symptom onset and had short disease survival. We also detected 4 different polymorphic variants (3'-untranslated region [UTR] variant, c. $* 41 \mathrm{G}>\mathrm{A}$; c.523+3ins[GAGGTG]; c.335-15del[TTTT]; and rs 13331793) in 9 patients from within our cohort. This study underlines the importance of population-based mutation screening of newly identified genes.
\end{abstract}

(C) 2011 Elsevier Inc. All rights reserved.

Keywords: ALS; FUS gene; Mutation; Sporadic

\section{Introduction}

Amyotrophic lateral sclerosis (ALS) is an adult-onset neurodegenerative disorder characterized by the loss of upper and lower motor neurons, resulting in progressive mus-

\footnotetext{
* Corresponding author at: Institute of Neurological Sciences, CNR, C. da Burga, Mangone 87050 (Cosenza), Italy. Tel.: + 390984 9801216; fax: +390984969306.

E-mail address: fl.conforti@isn.cnr.it (F.L. Conforti).
}

cle weakness and atrophy, which causes death within 3 to 5 years from symptom onset.

In the last decade several genes implicated in the RNA processing pathways have been associated with ALS, including the fused in sarcoma/traslocated in liposarcoma gene (FUS/TLS) (Strong, 2010). FUS is a ubiquitously expressed 526 amino acid protein, encoded by 15 exons, that belongs to the FET/TET family (FUS, EWS and TAF15 proto-oncoproteins) of multifunctional DNA/RNA binding proteins. FUS contains an N-terminal Gln-Gly-Ser-Tyr-rich domain, a Gly-rich domain, an RNA recognition motif, 
multiple Arg-Gly-Gly repeats, a zinc-finger motif and a highly conserved extreme C-terminus that encodes a nonclassic nuclear localization signal (NLS), recognized by transportin (Mackenzie et al., 2010).

Mutations in the FUS gene have been identified as the primary cause of ALS 6, an autosomal dominant form of familial ALS (FALS) linked to chromosome 16. The only exception is the Cape Verdean family for which the FUS mutation (p. H517Q) causes ALS only in a homozygous state (Lagier-Tourenne and Cleveland, 2009).

Subsequent reports of FUS mutations have also been described in sporadic patients (Talbot, 2009).

Much evidence suggests that mutations in FUS are associated with earlier onset of ALS than the general mean age of approximately 60 years (Corrado et al., 2010; Kwiatkowski et al., 2009; Vance et al., 2009).

In most cell types, FUS is present in both the nucleus and cytoplasm, but in neurons the proportion of FUS is higher in the nucleus than in the cytoplasm and in glia FUS is exclusively nuclear (Neumann et al., 2009). Neuropathological analysis of brain and spinal cord of ALS patients carrying mutations showed cytoplasmic retention and the formation of FUS ubiquitin-positive neuronal aggregates (DeJesusHernandez et al., 2010).

In only 2 years, 35 different pathogenic FUS mutations have been identified; they account for about $4 \%$ of familial ALS cases, but less than $1 \%$ of sporadic ALS cases. Most of these mutations (21 missense mutations) are clustered in the NLS region (encoded by exons 14 and 15) and may exert their pathogenic effects by disrupting the subcellular distribution of the protein, assuming a toxic gain of function effect (Hewitt et al., 2010; Mackenzie et al., 2010).

In this study, in order to investigate the presence and frequency of FUS mutations in our cohort of south Italian ALS patients, we performed a mutational screening of the FUS gene in 327 SOD1-negative, $A N G$-negative, $V A P B-$ negative, and TARDBP-negative sporadic ALS (SALS) cases. We also wished to report the clinical findings of those ALS patients carrying FUS mutations.

\section{Methods}

\subsection{Patients}

A total of 327 unrelated Caucasian patients from southern Italy (185 men and 142 women; mean age at onset 56.5 years, SD 12.2381) were included in this study. Each patient underwent a full neurological evaluation including electromyography, clinical laboratory testing, and imaging as appropriate to establish the clinical diagnosis of ALS according to the El Escorial criteria (Brooks et al., 2000). Patients were classified as sporadic when none of them had a known family history of ALS. Age at onset, gender distribution, site of symptom onset (bulbar and/or spinal-onset), predominance of upper/lower motor neuron signs, and disease duration were recorded (Table 1). Mini Mental State Exami-
Table 1

Clinical and genetic details of amyotrophic lateral sclerosis (ALS) patients

\begin{tabular}{ll}
\hline Sporadic ALS cases & $n=327$ \\
\hline Mean age at onset, y & 56.55 \\
Male $(\%)$ & $185(56.6)$ \\
Female $(\%)$ & $142(43.4)$ \\
Site of symptom onset $(\%)^{\mathrm{a}}$ & \\
$\quad$ Bulbar-onset disease & $55(17.2)$ \\
$\quad$ Spinal-onset disease & $261(81.6)$ \\
$\quad$ Lower & $125(47.9)$ \\
$\quad$ Upper & $122(46.7)$ \\
Mean disease duration $(\mathrm{mo})^{\mathrm{b}}$ & 42.03 \\
SOD1-, VAPB-, ANG-, TARDBP-positive & None
\end{tabular}

a Site of symptom onset data were not available for 7 cases.

b Disease duration data were not available for 40 cases.

nation (MMSE), Frontal Assessment Battery (FAB) and Beck Depression Inventory were administered in FUS mutation carriers to evaluate general and severe cognitive deterioration, frontal cognitive impairment, and severe depression, respectively.

All patients tested negative for mutations in $S O D 1, A N G$, $V A P B$, and TARDBP genes.

Control DNA was obtained from 100 unrelated, ethnically matched unaffected subjects with a negative personal and familial history for neurodegenerative diseases. Informed written consent was obtained from each individual, and appropriate institutional review board approval was obtained concerning human subjects. Blood samples were collected and DNA was extracted using standard protocols.

\subsection{Molecular analysis}

Until now, the previously reported variants, predicting an amino acid change, were all located in exons 3, 5, 6, 12, 14, and 15 , which are the only FUS regions affected by mutations in ALS patients; therefore, we specifically screened these exons in 327 SALS patients. Twelve primer pairs were designed from genomic DNA to amplify, by polymerase chain reaction (PCR), 6 coding exons and relative intron/ exon boundaries of the FUS gene. The amplicons for exons $3,5,6,12$, and 14 were analyzed by denaturing highperformance liquid chromatography (DHPLC) (Wave Transgenomic, Mountain View, CA, USA), while exon 15 was amplified and directly sequenced in each individual using the BigDye terminator v3.1 sequencing chemistry (Applied Biosystems, Foster City, CA, USA) and run on an ABI PRISM 3130 Genetic Analyzer (Applied Biosystem). In each case, forward primer or reverse primer was used for sequencing and variations were confirmed by sequencing an independent polymerase chain reaction product. Nucleotide numbering of FUS variations reflects cDNA numbering with +1 corresponding to the A of the ATG translation initiation codon reported in the GenBank reference sequence NM_004960.2, according to the journal guidelines (www.hgvs.org/mutnomen/). The initiation codon is codon 1. 


\subsection{Statistical analysis}

Statistical differences between SALS patients carrying FUS mutations and the whole ALS cohort were evaluated. In particular, because the expected frequencies were low, Fisher exact test and Monte Carlo method were used to compare gender distribution and site of onset, respectively. Age at onset and disease duration were evaluated using Mann-Whitney $U$ test.

\section{Results}

Molecular screening of the exons 3, 5, 6, 12, 14, and 15 of the FUS gene showed 3 different missense mutations in 4/327 SALS patients (Table 2). Two different substitutions occurring in the same amino acidic position have been observed in 2 patients: R521G and R521C respectively; P525L mutation has been found in 2 additional cases. All the observed mutations were found in exon 15 of the FUS gene and have been previously reported (Kwiatkowski et al., 2009; Ticozzi et al., 2009; Vance et al., 2009).

The mean of age at onset in these patients (38.75 years) was lower than in the whole cohort (56.77 years), and this difference is statistically significant (Mann-Whitney $U$ test, $p=0.016$ ). In all cases, except 1 , symptoms onset was before 50 years of age.

The neurological examination of the 4 FUS mutation carriers showed a muscle atrophy of the upper and lower limbs. Three out of the 4 FUS carriers also showed upper motor neuron signs. Electromyograms (EMGs) showed denervation in multiple myotomes of the upper and lower limbs, where motor unit potentials (MUPs) were polyphasic and increased in number and duration. Fibrillation potentials and fasciculations were also recorded. Motor and sensory conduction studies were within normal ranges. Cerebrospinal fluid analysis, levels of vitamins, thyroid function, and immunoelectrophoresis were normal. Brain and spine

Table 2

Clinical characteristics of FUS mutation carriers

\begin{tabular}{lllll}
\hline Patient & ALS-01 & ALS-02 & ALS-03 & ALS-04 \\
\hline Mutation & R521C & R521G & P525L & P525L \\
Exon & 15 & 15 & 15 & 15 \\
Gender & $\mathrm{F}$ & $\mathrm{M}$ & $\mathrm{M}$ & $\mathrm{F}$ \\
Age of onset, y & 53 & 32 & 26 & 45 \\
Death & Yes & No & Yes & Yes \\
Disease duration & 60 & 27 & 13 & 42 \\
$\quad$ (mo) & & & & \\
Site of onset & Upper & Upper & Lower & Upper \\
& limb & limb & limb & limb \\
Frontotemporal & No & No & No & No \\
$\quad$ cognitive & & & & \\
$\quad$ impairment & & & Yes & Yes \\
Bulbar signs & No & Yes & No & Yes \\
UMN signs & Yes & Yes & Yes & Yes \\
LMN signs & Yes & Yes &
\end{tabular}

Key: F, female; LMN, lower motor neuron; M, male, UMN, upper motor neuron. magnetic resonance imaging (MRI) scans were normal. None of the patients in this study showed signs of cognitive impairment, and all cases but 1 (ALS-03) showed a site of onset in the upper limb.

In detail, the patient carrying the R521C mutation (ALS01) was a 55-year-old married woman, who presented a 2-year history of progressive weakness and atrophy of the proximal muscles of the right upper limb. Her past medical history was negative. Her parents were not consanguineous. A clinical diagnosis of spinal-onset ALS was made. As the respiratory function progressively deteriorated, she underwent a tracheostomy. A few years later, the patient died of acute renal failure.

The patient carrying the R521G mutation (ALS-02) is a 34-year-old man who experienced onset at age 32 . He showed predominantly upper motor neuron signs with fasciculations and onset in the upper limbs. His parents were not available for the study. The patient is still alive.

One of the patients carrying the P525L mutation (ALS03 ) was a 26-year-old man, who had a 7-month history of progressive weakness and wasting. Weakness began in the lower limbs and progressed to the muscles of the upper limbs 5 months later. His past medical history was negative. His parents were not consanguineous and were not available for the study. A clinical diagnosis of lower motoneuron disease was made. One month later the patient died of acute cardiorespiratory failure.

The other patient carrying the P525L mutation (ALS-04) was a 45-year-old woman affected by multiple sclerosis with predominantly cerebellar and sensory disturbances, confirmed by neuroradiological and neuroimmunological investigations (intrathecal oligoclonal IgG synthesis). The patient developed a progressive weakness at the upper limbs (prominently at the left side) with diffuse fasciculations, intrinsic hand-muscle hypotrophy, followed by dysphagia, dysphonia, and progressive weakness of the 4 limbs. A diagnosis of ALS was made based on clinical and electrophysiological criteria. The patient underwent percutaneous endoscopic gastrostomy (PEG), and she died of respiratory failure 42 months after the onset of the disorder.

In our screening, we also found 4 different known polymorphic variants in 9 cases (Corrado et al., 2010; Ticozzi et al., 2009). In 6 patients we detected the $3^{\prime}$ untranslated region (UTR) variant, c. $* 41 \mathrm{G}>\mathrm{A}$, and in no cases was familial history of the disease recorded. We identified the variation c.1393+34G $>\mathrm{T}$ (rs13331793) in 1 female patient, who was diagnosed at 49 years of age with definite sporadic ALS, and the variation c.521_523+3delGAGGTG in the P525L-carrier patient described above (ALS-03). The known intronic variation c.335-15 del[TTTT] was detected in 1 previously described male patient (Conforti et al., 2004). Two of the above-mentioned polymorphic variants, c. $* 41 \mathrm{G}>\mathrm{A}$ and c.335-15 del[TTTT], were also observed in our control subjects with a frequency of $0.3 \%$ and $0.1 \%$, respectively. 


\section{Discussion}

In the current study, we reported a molecular screening of the FUS/TLS gene in 327 sporadic ALS patients from southern Italy. We detected 3 different missense mutations in 4 sporadic ALS cases (p. R521C, p. R521G, p. P525L) with a frequency of $1.25 \%$, which is slightly higher than what has been previously reported (Belzil et al., 2009; Corrado et al., 2010; Ticozzi et al., 2009). However, all patients must be considered apparently sporadic because either there was no family member DNA available for testing or there was a lack of information about the patients' family trees.

Regarding the clinical characteristics of the 4 FUS mutated patients (Table 2), the mean age at onset in these ALS patients was significantly lower (38.75 years) than in the whole cohort (56.78). This result confirms that mutations in FUS are associated with an earlier onset of the disease in comparison with the general mean age of approximately 60 years reported for ALS (Corrado et al., 2010).

None of the patients in this study showed signs of cognitive impairment. Approximately 5\% of patients with ALS also develop frontotemporal dementia (Groen et al., 2010), and to date only a small number of ALS/dementia patients with FUS mutations have been described, but it seems that cognitive dysfunction is absent or rare in FUSmediated ALS (Blair et al., 2009; Groen et al., 2010; Lagier-Tourenne and Cleveland, 2009; Ticozzi et al., 2009; Yan et al., 2010).

The mutations p.R521C and p.R521G were observed in 2 sporadic ALS cases (ALS-01, ALS-02) without an apparent family history of motoneuron disease. The arginine at codon 521 is preserved during evolution, and it represents the most commonly mutated amino acid, identified in 11 families and in 2 sporadic cases (Rademakers et al., 2010). Both amino acid substitutions disrupt the NLS and lead to an aberrant subcellular distribution of FUS protein, with retention in the cytoplasm. In literature, the mutation $\mathrm{R} 521 \mathrm{G}$ has been described with a reduced penetrance of the disease (Kwiatkowski et al., 2009); while mutation R521C has been associated with complete penetrance (Corrado et al., 2010). Even if both of these mutations occur in the same codon position and possibly cause the same cellular damage, patients ALS-01 (R521G carrier) and ALS-02 (R521C carrier) showed different clinical pictures for age of onset and disease course. Indeed, patient ALS-01 had an onset at 53 years and showed a survival duration that was much longer than ALS-02 patient (Table 2). The patient carrying the p.R521C mutation showed a clinical phenotype similar to that already described in Italian patients (Corrado et al., 2010; Ticozzi et al., 2009). This confirms the possibility to associate specific phenotypes with some FUS mutations, even if the identification of additional patients carrying the same mutation should provide more insight into the genotype-phenotype correlations.
The mutation p.P525L, which also localized in exon 15 of the gene, was detected in 2 SALS patients (ALS-03 and ALS-04). All previously described p.P525L mutation carriers showed an aggressive course of the disease and no family history has been documented in any previous studies (Bäumer et al., 2010; Chiò et al., 2009; Kwiatkowski et al., 2009). The clinical pattern observed in patient ALS-03 (young age at onset at 26 years and rapid progression causing death within 12 months), is similar to that already reported for this mutation, confirming that the p.P525L substitution results in a highly aggressive form of ALS. Unfortunately, DNA samples from the unaffected parents were not available, and this patient must be considered apparently sporadic as well. Furthermore, this patient carried both the P525L mutation and the known variation c.521_523+3delGAGGTG in exon5. This deletion was first reported as a pathogenic mutation in FALS patients (Kwiatkowski et al., 2009) and was also detected in healthy subjects (Rademakers et al., 2010). These findings, together with our data further suggest that it does not represent a pathogenic mutation.

Instead, a different course of the disease was observed in the other p.P525L carrier, ALS-04, who showed a demyelinating disease associated with SALS. The pathological evidence of both ALS and multiple sclerosis in a FUS mutation carrier was also reported in a British familial ALS patient (Hewitt et al., 2010). In our patient, the demyelinating disease was already present at the age of 45, when ALS symptoms arose and led to death of the patient after 42 months, a much longer period of time than any other case described. Obviously, the onset variability and survival duration seen in the patients carrying the same mutation, could be attributed to additional genetic and/or environmental factors, which could modify the penetrance of the disease.

In conclusion, in our cohort of 327 SALS patients we detected 4 FUS mutations, representing $1.25 \%$ of SALS cases, and the occurrence of several polymorphisms. Moreover, although our data illustrates that patients with FUS mutations may develop either classic or atypical ALS phenotypes, it seems that most patients predominantly demonstrate earlier symptom onset and short disease survival (Yan et al., 2010). Furthermore, this study highlights the importance of population-based mutation screening of newly identified genes, such as $F U S$, as a starting point for investigating the pathogenic mechanism leading to ALS.

\section{Disclosure statement}

None of the authors has actual or potential conflicts of interest.

Informed written consent was obtained from each individual, and appropriate institutional review board approval was obtained concerning human subjects. 


\section{Acknowledgements}

The authors' research on ALS genetics is funded by the National Research Council, Institute of Neurological Sciences.

\section{References}

Bäumer, D., Hilton, D., Paine, S.M., Turner, M.R., Lowe, J., Talbot, K., Ansorge, O., 2010. Juvenile ALS with basophilic inclusions is a FUS proteinopathy with FUS mutations. Neurology 75, 611-618.

Belzil, V.V., Valdmanis, P.N., Dion, P.A., Daoud, H., Kabashi, E., Noreau, A., Gauthier, J., S2D team, Hince, P., Desjarlais, A., Bouchard, J.P., Lacomblez, L., Salachas, F., Pradat, P.F., Camu, W., Meininger, V., Dupré, N., Rouleau, G.A., 2009. Mutations in FUS cause FALS and SALS in French and French Canadian populations. Neurology 73, 1176-1179.

Blair, I.P., Williams, K.L., Warraich, S.T., Durnall, J.C., Thoeng, A.D., Manavis, J., Blumbergs, P.C., Vucic, S., Kiernan, M.C., Nicholson, G.A., 2009. FUS mutations in amyotrophic lateral sclerosis: clinical, pathological, neurophysiological and genetic analysis. J. Neurol. Neurosurg. Psychiatry 81, 639-641.

Brooks, B.R., Miller, R.G., Swash, M., Munsat, T.L., 2000. World Federation of Neurology Research Group on Motor Neuron Diseases. El Escorial revisited: revised criteria for the diagnosis of amyotrophic lateral sclerosis. Amyotroph. Lateral Scler. Other Mot. Neuron Disord. 1, 293-299.

Chiò, A., Restagno, G., Brunetti, M., Ossola, I., Calvo, A., Mora, G., Sabatelli, M., Monsurrò, M.R., Battistini, S., Mandrioli, J., Salvi, F., Spataro, R., Schymick, J., Traynor, B.J., La Bella, V., ITALSGEN Consortium, 2009. Two Italian kindreds with familial amyotrophic lateral sclerosis due to FUS mutation. Neurobiol. Aging 30, 12721275

Conforti, F.L., Magariello, A., Mazzei, R., Sprovieri, T., Patitucci, A., Crescibene, L., Bastone, L., Gabriele, A., Scornaienchi, M., Ferraro, T., Muglia, M., Quattrone, A., 2004. Abnormally high levels of SOD1 mRNA in a patient with amyotrophic lateral sclerosis. Muscle Nerve $29,610-611$.

Corrado, L., Del Bo, R., Castellotti, B., Ratti, A., Cereda, C., Penco, S., Sorarù, G., Carlomagno, Y., Ghezzi, S., Pensato, V., Colombrita, C., Gagliardi, S., Cozzi, L., Orsetti, V., Mancuso, M., Siciliano, G., Mazzini, L., Comi, G.P., Gellera, C., Ceroni, M., D’Alfonso, S., Silani, V., 2010. Mutations of FUS gene in sporadic amyotrophic lateral sclerosis. J. Med. Genet. 47, 190-194.

DeJesus-Hernandez, M., Kocerha, J., Finch, N., Crook, R., Baker, M. Desaro, P., Johnston, A., Rutherford, N., Wojtas, A., Kennelly, K., Wszolek, Z.K., Graff-Radford, N., Boylan, K., Rademakers, R., 2010. De novo truncating FUS gene mutation as a cause of sporadic amyotrophic lateral sclerosis. Hum. Mutat. 31, E1377-E1389.
Groen, E.J., van Es, M.A., van Vught, P.W., Spliet, W.G., van EngelenLee, J., de Visser, M., Wokke, J.H., Schelhaas, H.J., Ophoff, R.A., Fumoto, K., Pasterkamp, R.J., Dooijes, D., Cuppen, E., Veldink, J.H., van den Berg, L.H., 2010. FUS mutations in familial amyotrophic lateral sclerosis in the Netherlands. Arch. Neurol. 67, 224-230.

Hewitt, C., Kirby, J., Highley, J.R., Hartley, J.A., Hibberd, R., Hollinger, H.C., Williams, T.L., Ince, P.G., McDermott, C.J., Shaw, P.J., 2010. Novel FUS/TLS mutations and pathology in familial and sporadic amyotrophic lateral sclerosis. Arch. Neurol. 67, 455-461.

Kwiatkowski, T.J., Jr., Bosco, D.A., Leclerc, A.L., Tamrazian, E., Vanderburg, C.R., Russ, C., Davis, A., Gilchrist, J., Kasarskis, E.J., Munsat, T., Valdmanis, P., Rouleau, G.A., Hosler, B.A., Cortelli, P., de Jong, P.J., Yoshinaga, Y., Haines, J.L., Pericak-Vance, M.A., Yan, J., Ticozzi, N., Siddique, T., McKenna-Yasek, D., Sapp, P.C., Horvitz, H.R., Landers, J.E., Brown, R.H., Jr., 2009. Mutations in the FUS/TLS gene on chromosome 16 cause familial amyotrophic lateral sclerosis. Science 323, 1205-1208.

Lagier-Tourenne, C., Cleveland, D.W., 2010. Rethinking ALS: the FUS about TDP-43. Cell 136, 1001-1004.

Mackenzie, I.R., Rademakers, R., Neumann, M., 2010. TDP-43 and FUS in amyotrophic lateral sclerosis and frontotemporal dementia. Lancet Neurol. 9, 995-1007.

Neumann, M., Roeber, S., Kretzschmar, H.A., Rademakers, R., Baker, M., Mackenzie, I.R., 2009. Abundant FUS-immunoreactive pathology in neuronal intermediate filament inclusion disease. Acta Neuropathol. 118, 605-616.

Rademakers, R., Stewart, H., Dejesus-Hernandez, M., Krieger, C., GraffRadford, N., Fabros, M., Briemberg, H., Cashman, N., Eisen, A., Mackenzie, I.R., 2010. Fus gene mutations in familial and sporadic amyotrophic lateral sclerosis. Muscle Nerve 42, 170-176.

Strong, M.J., 2010. The evidence for altered RNA metabolism in amyotrophic lateral sclerosis (ALS). J. Neurol. Sci. 288, 1-12.

Talbot, K., 2009. Another gene for ALS: mutations in sporadic cases and the rare variant hypothesis. Neurology 73, 1172-1173.

Ticozzi, N., Silani, V., LeClerc, A.L., Keagle, P., Gellera, C., Ratti, A., Taroni, F., Kwiatkowski, T.J., Jr., McKenna-Yasek, D.M., Sapp, P.C., Brown, R.H., Jr., Landers, J.E., 2009. Analysis of FUS gene mutation in familial amyotrophic lateral sclerosis within an Italian cohort. Neurology 73, 1180-1185.

Vance, C., Rogelj, B., Hortobágyi, T., De Vos, K.J., Nishimura, A.L., Sreedharan, J., Hu, X., Smith, B., Ruddy, D., Wright, P., Ganesalingam, J., Williams, K.L., Tripathi, V., Al-Saraj, S., Al-Chalabi, A., Leigh, P.N., Blair, I.P., Nicholson, G., de Belleroche, J., Gallo, J.M., Miller, C.C., Shaw, C.E., 2009. Mutations in FUS, an RNA processing protein, cause familial amyotrophic lateral sclerosis type 6 . Science 323, 1208-1211.

Yan, J., Deng, H.X., Siddique, N., Fecto, F., Chen, W., Yang, Y., Liu, E., Donkervoort, S., Zheng, J.G., Shi, Y., Ahmeti, K.B., Brooks, B., Engel, W.K., Siddique, T., 2010. Frameshift and novel mutations in FUS in familial amyotrophic lateral sclerosis and ALS/dementia. Neurology $75,807-814$. 